N. Tanaka

Nagoya Math. J.

Vol. 51 (1973), 137-160

\title{
RIGIDITY FOR ELLIPTIC ISOMETRIC IMBEDDINGS
}

\author{
NOBORU TANAKA
}

\section{Introduction}

The main purpose of the present paper is to give the details of the results announced in the $\mathrm{P}$. J. A. note [11], establishing some global theorems on rigidity for a certain class of isometric imbeddings.

We first introduce the notion of an elliptic imbedding: An imbedding $\boldsymbol{f}$ of a manifold $M$ in a Euclidean space $\boldsymbol{R}^{m}$ is called elliptic if it is generic in a suitable sense and if the second fundamental form corresponding to any normal vector $\neq 0$ of the imbedding has at least two eigenvalues of the same sign. We then prove a rigidity theorem (Theorem 2.4) which may be roughly stated as follows: Let $f_{0}$ be an imbedding of $M$ in $\boldsymbol{R}^{m}$. Assume that 1) $f_{0}$ is elliptic, 2) it is "infinitesimally rigid" and 3) $M$ is compact. If two imbeddings $f$ and $\boldsymbol{f}^{\prime}$ of $M$ in $\boldsymbol{R}^{m}$ lie both near to $f_{0}$ with respect to the $C^{3}$-topology and if they induce the same Riemannian metric on $M$, then there is a unique Euclidean transformation $a$ of $\boldsymbol{R}^{m}$ such that $\boldsymbol{f}^{\prime}=a \boldsymbol{f}$. Moreover we apply Theorem 2.4 to the canonical isometric imbedding $f_{0}$ of a compact hermitian symmetric space $M=G / H$ in the Euclidean space $\boldsymbol{R}^{m}$, where $m=\operatorname{dim} G$. In fact it is shown that the imbedding $\boldsymbol{f}_{0}$ satisfies the conditions 1), 2) and 3) stated above (Proposition 3.3 and Theorem 3.4). Thus we obtain a rigidity theorem (Theorem 3.5) for imbeddings around $f_{0}$, which turns out to be a partial generalization of the famous theorem of Cohn-Vossen.

In $\S 1$ we first define an important differential operator $L=L^{f}$, which is associated with every imbedding $f$ of $M$ in $R^{m}$ satisfying condition $(C)$, where "condition $(C)$ " is the very generality condition to impose on an elliptic imbedding. It is shown that the operator $L$ is, in a suitable sense, equivalent to the differential operator $\Phi_{* f}$, "the operator of infinitesimal isometric deformations" of $f$ (Theorem 1.2). We then

Received December 25, 1972. 
proceed to the definition of an elliptic imbedding and find that an imbedding $\boldsymbol{f}$ is elliptic if and only if the operator $L$ is elliptic.

In $\S 2$ we prove Theorem 2.4. The proof heavily depends on Theorem 2.3 which is the principle of upper semi-continuity concerning the operators $L^{f}$ parametrized by the elliptic imbeddings $f$ and which is analogous to Theorem 4 in Kodaira-Spencer [4]. Finally $\S 3$ is devoted to the proof of Theorem 3.4, which needs some calculations on the Laplacian $\Delta$ of the hermitian symmetric space $M=G / H$.

\section{Preliminary remark}

Throughout the present paper we shall always assume the differentiability of class $C^{\infty}$.

Let $E$ be a differentiable vector bundle over a differentiable manifold $M . \quad E_{p}$ will denote the fibre of $E$ over a point $p \in M . \Gamma(E)$ will denote the vector space of differentiable cross sections on $M$. An inner product $\langle$,$\rangle in E$ is an assignment which assigns to every point $p \in M$ an inner product $\langle$,$\rangle in the fibre E_{p}$ and which is differentiable in an appropriate sense.

\section{§1. Elliptic imbeddings}

1.1. The differential operator $\Phi_{* f}$. Let $M$ be a connected differentiable manifold. $T$ denotes the tangent bundle of $M$ and $T^{*}$ its dual. $S^{2} T^{*}$ denotes the vector bundle of symmetric tensors of type $\left(\begin{array}{l}0 \\ 2\end{array}\right)$ on $M$. For $\alpha, \beta \in T_{p}^{*}, \alpha \cdot \beta$ denotes the symmetric product of $\alpha$ and $\beta$, being an element of $S^{2} T_{p}^{*}$.

Let $\boldsymbol{R}^{m}$ be the space of $m$ real variables and $x_{1}, \cdots, x_{m}$ the canonical coordinates of it. As usual $\boldsymbol{R}^{m}$ is an $m$-dimensional Euclidean space (flat Riemannian manifold) with respect to the Riemannian metric $d s^{2}=$ $\sum_{i} d x_{i}^{2} . \quad \boldsymbol{R}^{m}$ is also considered as an $m$-dimensional Euclidean vector space with respect to the inner product $\langle$,$\rangle defined as follows: \langle\boldsymbol{a}, \boldsymbol{b}\rangle=$ $\sum_{i} a_{i} b_{i}$, where $\boldsymbol{a}=\left(a_{1}, \cdots, a_{m}\right), \boldsymbol{b}=\left(b_{1}, \cdots, b_{m}\right) \in \boldsymbol{R}^{m}$.

Let $\boldsymbol{\alpha}=\left(\alpha_{1}, \cdots, \alpha_{m}\right)$ and $\beta=\left(\beta_{1}, \cdots, \beta_{m}\right)$ be $\boldsymbol{R}^{m}$-valued 1-forms on $M$ and let $\boldsymbol{f}=\left(f_{1}, \cdots, f_{m}\right)$ be an $\boldsymbol{R}^{m}$-valued function on $M .\langle\boldsymbol{\alpha}, \boldsymbol{\beta}\rangle$ denotes the cross section of $S^{2} T^{*}$ defined by $\langle\boldsymbol{\alpha}, \boldsymbol{\beta}\rangle=\sum_{i} \alpha_{i} \beta_{i}$ or

$$
\langle\boldsymbol{\alpha}, \boldsymbol{\beta}\rangle(X, Y)=\frac{1}{2}(\langle\boldsymbol{\alpha}(X), \beta(Y)\rangle+\langle\boldsymbol{\alpha}(Y), \beta(X)\rangle) .
$$

for all $X, Y \in T_{p}$ and $p \in M$. $\langle\boldsymbol{f}, \boldsymbol{\alpha}\rangle$ denotes the 1-form on $M$ defined by 
$\langle\boldsymbol{f}, \boldsymbol{\alpha}\rangle=\sum_{i} f_{i} \alpha_{i}$ or $\langle\boldsymbol{f}, \boldsymbol{\alpha}\rangle(X)=\langle\boldsymbol{f}, \boldsymbol{\alpha}(X)\rangle$. $d \boldsymbol{f}$ denotes the exterior derivative of $\boldsymbol{f}: d \boldsymbol{f}=\left(d f_{1}, \cdots, d f_{m}\right)$.

Let $\Gamma(M, m)$ be the vector space of differentiable maps of $M$ to $\boldsymbol{R}^{m}$, which may be regarded as the vector space of $\boldsymbol{R}^{m}$-valued differentiable functions on $M$. Let $\subseteq(M, m)$ be the subset of $\Gamma(M, m)$ consisting of all the imbeddings of $M$ in $\boldsymbol{R}^{m}$. We assume $\mathfrak{F}(M, m) \neq \phi$. For $\boldsymbol{f} \in \mathbb{E}(M, m)$, we denote by $\Phi(f)$ the Riemannian metric $f^{*} d s^{2}$ on $M$ which is induced from $d s^{2}$ by the imbedding $\boldsymbol{f}$; We have

$$
\Phi(\boldsymbol{f})=\langle d \boldsymbol{f}, d \boldsymbol{f}\rangle .
$$

Given a Riemannian metric $\nu$ on $M, f \in \mathbb{E}(M, m)$ is called an isometric imbedding of the Riemannian manifold $(M, \nu)$ in the Euclidean space $\boldsymbol{R}^{m}$ if $\Phi(f)$ coincides with the given $\nu$.

The assignment $\boldsymbol{f} \rightarrow \Phi(f)$ gives a map $\Phi$ of the set $\mathfrak{E}(M, m)$ to the set $\Re(M)$ of Riemannian metrics on $M$. For $f \in \mathfrak{F}(M, m)$, we define a differential operator $\Phi_{* f}$ of $\Gamma(M, m)$ to $\Gamma\left(S^{2} T^{*}\right)$, the differential of the map $\Phi$ at $f$, by

$$
\Phi_{* f}(\boldsymbol{u})=2\langle d \boldsymbol{f}, d \boldsymbol{u}\rangle
$$

for all $\boldsymbol{u} \in \Gamma(M, m)$.

1.2. The second fundamental forms. Let $f$ be an imbedding of $M$ in $\boldsymbol{R}^{m}$. Let $T^{\prime}$ be the vector bundle on $M$ which is induced from the tangent bundle $T\left(\boldsymbol{R}^{m}\right)$ of $\boldsymbol{R}^{m}$ by the imbedding $\boldsymbol{f}$. The Riemannian metric $d s^{2}$ on $\boldsymbol{R}^{m}$ induces an inner product in the vector bundle $T^{\prime}$ and the tangent bundle $T$ of $M$ may be identified with a subbundle of $T^{\prime}$. This being said, the normal bundle $N$ of $\boldsymbol{f}$ is the orthogonal complement of $T$ in $T^{\prime}$ with respect to the inner product in $T^{\prime}: T^{\prime}=T \oplus N$. $T^{\prime}$ being a trivial bundle in a canonical manner, every fibre $T_{p}^{\prime}$ of $T^{\prime}$ may be identified with the Euclidean vector space $\boldsymbol{R}^{m}$ and hence a cross section of $T^{\prime}$ may be regarded as an $\boldsymbol{R}^{m}$-valued function on $M$ and vice versa.

Let $\nabla$ be the covariant differentiation ${ }^{1)}$ (Riemannian connection) associated with the Riemannian metric $\nu=\Phi(f)$. Given an $\boldsymbol{R}^{m}$-valued tensor field $\boldsymbol{a}=\left(\alpha_{1}, \cdots, \alpha_{m}\right)$ of type $\left({ }_{p}^{0}\right)$ on $M$, we define the covariant derivative $\nabla \boldsymbol{\alpha}$ of $\boldsymbol{\alpha}$ in an obvious manner: $\nabla \boldsymbol{\alpha}=\left(\nabla \alpha_{1}, \cdots, \nabla \alpha_{m}\right)$. Note that $\nabla_{X} \boldsymbol{u}=d \boldsymbol{u}(X)=X \boldsymbol{u}$ for all $X \in T_{p}$ and $\boldsymbol{R}^{m}$-valued functions $\boldsymbol{u}$ on $M$.

The following proposition is known.

\footnotetext{
1) As for the covariant differentiation $\nabla$, we use the same notations as in [3].
} 
Proposition 1.1. For all $X, Y \in T_{p}$, the vectors $\nabla_{X} \nabla_{Y} f$ are in the fibre $N_{p}$ of the normal bundle $N$.

Proof. We have $\left\langle\nabla_{Y} f, \nabla_{Z} f\right\rangle=\nu(Y, Z)$ for all $Y, Z \in T_{p}$, whence

$$
\left\langle\nabla_{X} \nabla_{Y} f, \nabla_{Z} \boldsymbol{f}\right\rangle+\left\langle\nabla_{Y} \boldsymbol{f}, \nabla_{X} \nabla_{Z} \boldsymbol{f}\right\rangle=\left(\nabla_{X} \nu\right)(Y, Z)=0
$$

For al! $X \in T_{p}$. Hence we have

$$
\begin{gathered}
\left\langle\nabla_{Y} \nabla_{X} \boldsymbol{f}, \nabla_{X} \boldsymbol{f}\right\rangle=0, \\
\left\langle\nabla_{X} \nabla_{X} \boldsymbol{f}, \nabla_{Y} \boldsymbol{f}\right\rangle+\left\langle\nabla_{X} \boldsymbol{f}, \nabla_{X} \nabla_{Y} \boldsymbol{f}\right\rangle=0
\end{gathered}
$$

for all $X, Y \in T_{p}$. Since $\nabla_{X} \nabla_{Y} f=\nabla_{Y} \nabla_{X} f$ and since $T_{p}$ is composed of the vectors $\nabla_{Y} f\left(Y \in T_{p}\right)$, we have $\nabla_{X} \nabla_{X} f \in N_{p}$ and hence $\nabla_{X} \nabla_{Y} f \in N_{p}$, proving our assertion.

Let us now consider the following condition $(C)^{2)}$ for the imbedding $\boldsymbol{f}$ : At each $p \in M$, the fibre $N_{p}$ of $N$ is spanned by the vectors of the form $\nabla_{X} \nabla_{Y} f$, where $X, Y \in T_{p}$.

For $\boldsymbol{a} \in N_{p}$, we define an element $\theta_{\boldsymbol{a}}$ of $S^{2} T_{p}^{*}$ by

$$
\theta_{\boldsymbol{a}}(X, Y)=\left\langle\boldsymbol{a}, \nabla_{X} \nabla_{Y} \boldsymbol{f}\right\rangle,
$$

which is usually called the second fundamental form of $f$ corresponding to the normal vector $a$. Then we see that $f$ satisfies condition $(C)$ if and only if the map $\theta: N \ni \boldsymbol{a} \rightarrow \theta_{\boldsymbol{a}} \in S^{2} T^{*}$ is injective.

1.3. The differential operator $L$. In what follows we assume that the imbedding $f$ satisfies condition $(C)$. By the above remark the image $\tilde{N}$ of $N$ by $\theta$ forms a subbundle of $S^{2} T^{*}$, which will be called the bundle of second fundamental forms of $\boldsymbol{f}$.

We define a differential operator $D$ of $\Gamma\left(T^{*}\right)$ to $\Gamma\left(S^{2} T^{*}\right)$ by

$$
(D \varphi)(X, Y)=\left(\nabla_{X} \varphi\right)(Y)+\left(\nabla_{Y} \varphi\right)(X)
$$

for all $\varphi \in \Gamma\left(T^{*}\right)$ and $X, Y \in T_{p}$, and denote by $\pi$ the projection of $S^{2} T^{*}$ onto the factor bundle $S^{2} T^{*} / \tilde{N}$. Then the composition $L=\pi \circ D$ is a differential operator of $\Gamma\left(T^{*}\right)$ to $\Gamma\left(S^{2} T^{*} / \tilde{N}\right)$.

THEOREM 1.2. Let $\boldsymbol{f}$ be an imbedding of $M$ in $\boldsymbol{R}^{m}$ satisfying condition (C) and let $\alpha \in \Gamma\left(S^{2} T^{*}\right)$. Then the solutions $\boldsymbol{u}$ of the equation

2) This is equivalent to the condition that, at each $p \in M$, the vector space $\boldsymbol{R}^{m}$ is spanned by the vectors of the form $\left(\partial f / \partial x_{i}\right)(p),\left(\partial^{2} f / \partial x_{i} \partial x_{j}\right)(p)$, where $x_{1}, \cdots, x_{n}$ is a coordinate system of $M$ at $p$. 
$\Phi_{* f}(\boldsymbol{u})=\alpha$ are in a one-to-one correspondence with the solutions $\varphi$ of the equation $L \varphi=\pi \alpha$, and the correspondence $\boldsymbol{u} \rightarrow \varphi$ is given by the relation $\varphi=\langle\boldsymbol{u}, d \boldsymbol{f}\rangle$.

Proof. Let $\boldsymbol{u} \in \Gamma(M, m)$. Put $\varphi=\langle\boldsymbol{u}, d \boldsymbol{f}\rangle$ and denote by $X$ the vector field on $M$ which is dual to the 1 -form $\varphi$ with respect to the Riemannian metric v. Put

$$
\boldsymbol{v}=\boldsymbol{u}-X \boldsymbol{f}
$$

Then we have

$$
\begin{aligned}
\langle\boldsymbol{v}, d \boldsymbol{f}(Y)\rangle & =\langle\boldsymbol{u}, d \boldsymbol{f}(Y)\rangle-\langle d \boldsymbol{f}(X), d \boldsymbol{f}(Y)\rangle \\
& =\varphi(Y)-\nu(X, Y)=0
\end{aligned}
$$

for all $Y \in T_{p}$, showing that $v$ may be regarded as a cross section of $N$. Furthermore from (1.1) we get

$$
\nabla_{Y} \boldsymbol{u}=\nabla_{Y} \boldsymbol{v}+\nabla_{Y} \nabla_{X} f+\left(\nabla_{Y} X\right) \boldsymbol{f}
$$

for all $Y \in T_{p}$. Since $\left\langle\boldsymbol{v}, \nabla_{Z} f\right\rangle=0$ for all $Z \in T_{p}$, we have $\left\langle\nabla_{Y} \boldsymbol{v}, \nabla_{Z} f\right\rangle=$ $-\left\langle\boldsymbol{v}, \nabla_{Y} \nabla_{Z} f\right\rangle$ for all $Y, Z \in T_{p}$. Therefore it follows from (1.2) and Proposition 1.1 that

$$
\begin{aligned}
\left\langle\nabla_{Y} \boldsymbol{u}, \nabla_{Z} \boldsymbol{f}\right\rangle & =\left\langle\left(\nabla_{Y} X\right) \boldsymbol{f}, \nabla_{Z} \boldsymbol{f}\right\rangle+\left\langle\nabla_{Y} \boldsymbol{v}, \nabla_{Z} \boldsymbol{f}\right\rangle \\
& =\nu\left(\nabla_{Y} Y, Z\right)-\left\langle\boldsymbol{v}, \nabla_{Y} \nabla_{Z} \boldsymbol{f}\right\rangle
\end{aligned}
$$

and hence

$$
\left\langle\nabla_{Y} \boldsymbol{u}, \nabla_{Z} \boldsymbol{f}\right\rangle=\left(\nabla_{Y} \varphi\right)(Z)-\theta_{\boldsymbol{v}}(Y, Z),
$$

where $\theta_{v}$ is the cross section of $\tilde{N}$ defined by $\left(\theta_{v}\right)_{p}=\theta(\boldsymbol{v}(p))$. Commuting $Y$ and $Z$ in the above equality, we also obtain

$$
\left\langle\nabla_{Z} \boldsymbol{u}, \nabla_{Y} \boldsymbol{f}\right\rangle=\left(\nabla_{Z} \varphi\right)(Y)-\theta_{\boldsymbol{v}}(Z, Y) .
$$

Therefore from these two equalities we get

$$
2\langle d \boldsymbol{f}, d \boldsymbol{u}\rangle=D \varphi-2 \theta_{\boldsymbol{v}} .
$$

Now suppose that $\boldsymbol{u}$ is a solution of the equation $\Phi_{* f}(\boldsymbol{u})=\alpha . \quad \theta_{v}$ being a cross section of $\tilde{N}$, we know from (1.3) that $\varphi$ is a solution of the equation $L \varphi=\pi \alpha$. Conversely let $\varphi$ be a solution of the equation $L \varphi=$ $\pi \alpha$. Since $D \varphi-\alpha$ is a cross section of $\tilde{N}$ and since $\theta: N \rightarrow \tilde{N}$ is an isomorphism, there is a unique cross section $\boldsymbol{v}$ of $N$ such that $D \varphi-\alpha$ $=2 \theta_{v}$. If we put $\boldsymbol{u}=\boldsymbol{v}+X \boldsymbol{f}, X$ being the dual to $\varphi$, then we can see 
from the above argument that $\langle\boldsymbol{u}, d \boldsymbol{f}\rangle=\varphi$ and that $\boldsymbol{u}$ is a solution of the equation $\Phi_{* f}(\boldsymbol{u})=\alpha$. We have thereby proved Theorem 1.2.

1.4. An elliptic imbedding. Let $\boldsymbol{f}$ be an imbedding of $M$ in $\boldsymbol{R}^{m}$ satisfying condition $(C)$. Let $\xi$ be any covector at any point $p \in M$, i.e., $\xi \in T_{p}^{*}$. The symbol $\sigma(\xi)$ of the operator $L$ at $\xi$ is a linear map of $T_{p}^{*}$ to $S^{2} T_{p}^{*} / \tilde{N}_{p}$ defined as follows: Take a function $f$ on $M$ such that $f(p)=0$ and $d f_{p}=\xi$ and, for any $\eta \in T_{p}^{*}$, take $\varphi \in \Gamma\left(T^{*}\right)$ such that $\varphi_{p}=\eta$. Then $\sigma(\xi) \eta=L(f \varphi)_{p}$. We have

$$
D(f \varphi)(X, Y)=\nabla_{X} f \cdot \varphi(Y)+\nabla_{Y} f \cdot \varphi(X)+f D \varphi(X, Y)
$$

for all $X, Y \in T_{p}$, whence $D(f \varphi)_{p}=2 \xi \cdot \eta$. Therefore we get

$$
\sigma(\xi) \eta=2 \pi(\xi \cdot \eta) \text {. }
$$

The operator $L$ is called elliptic if the symbol $\sigma(\xi)$ is injective at any non-zero covector $\xi$. (For the theory of elliptic linear differential operators on manifolds, we refer to [10].) Furthermore we shall say that a subbundle of $S^{2} T^{*}$ is elliptic if it contains no non-zero elements of the form $\xi \cdot \eta$, where $\xi$ and $\eta$ are covectors with the same origin, or equivalently if every symmetric form $(\neq 0)$ in the subbundle has at least two eigenvalues of the same sign.

By (1.4) we have the following

Proposition 1.3. The differential operator $L$ associated with $f$ is elliptic if and only if the bundle $\tilde{N}$ of second fundamental forms of $\boldsymbol{f}$ is elliptic.

We shall say that an imbedding $f$ of $M$ in $\boldsymbol{R}^{m}$ is elliptic if it satisfies condition $(C)$ and if the bundle $\tilde{N}$ is elliptic.

Proposition 1.4. Let $\boldsymbol{f}$ be an imbedding of $M$ in $\boldsymbol{R}^{m}$. We assume that $f$ is elliptic and that $M$ is compact. Then the solution space of the equation $\Phi_{* \boldsymbol{f}}(\boldsymbol{u})=0$ is finite dimensional.

This follows immediately from Theorem 1.2 and a well known theorem on elliptic operators.

\section{§ 2. A rigidity theorem for elliptic imbeddings}

2.1. Imbeddings and Euclidean transformations. Let $O(m)$ be the orthogonal group of degree $m$. A Euclidean transformation of $\boldsymbol{R}^{m}$ is a transformation $a$ of the form: 


$$
a \boldsymbol{x}=b \boldsymbol{x}+\boldsymbol{c} \quad\left(\boldsymbol{x} \in \boldsymbol{R}^{m}\right),
$$

where $b \in O(m)$ and $\boldsymbol{c} \in \boldsymbol{R}^{m}$. The set $E(m)$ of Euclidean transformations forms a Lie group.

Let $\mathfrak{o}(m)$ be the Lie algebra of skew-symmetric matrices of degree $m$, being the Lie algebra of $O(m)$. An infinitesimal Euclidean transformation of $\boldsymbol{R}^{m}$ is a map $A$ of $\boldsymbol{R}^{m}$ to itself of the form:

$$
A \boldsymbol{x}=B \boldsymbol{x}+\boldsymbol{c} \quad\left(\boldsymbol{x} \in \boldsymbol{R}^{n}\right),
$$

where $B \in \mathfrak{D}(m)$ and $\boldsymbol{c} \in \boldsymbol{R}^{m}$. The set $e(m)$ of infinitesimal Euclidean transformations forms a Lie algebra, being the Lie algebra of $E(m)$.

Let $M$ and $\boldsymbol{R}^{m}$ be as in $\S 1$. For $\boldsymbol{f} \in \mathfrak{S}(M, m)$ and $a \in E(m)$, we have $a \boldsymbol{f} \in \mathfrak{S}(M, m)$ and

$$
\Phi(a \boldsymbol{f})=\Phi(\boldsymbol{f}) .
$$

Thus the group $E(m)$ acts on $\mathbb{E}(M, m)$ as a transformation group and the map $\Phi$ is an invariant. It is clear that $E(m)$ leaves invariant the subset $\mathscr{F}_{0}$ of $\mathfrak{E}(M, m)$ composed of all the imbeddings satisfying condition $(C)$ as well as the subset $\mathfrak{F}_{1}$ of $\mathfrak{F}(M, m)$ composed of all the elliptic imbeddings.

Proposition 2.1. The group $E(m)$ freely acts on the subset $\mathfrak{F}_{0}$ of Es $(M, m)$.

Proof. Suppose that $a \boldsymbol{f}=\boldsymbol{f}$, where $a \in E(m)$ and $\boldsymbol{f} \in \mathfrak{F}_{0}$. Then $b \boldsymbol{f}+$ $\boldsymbol{c}=\boldsymbol{f}$, whence $b \nabla_{X} \boldsymbol{f}=\nabla_{X} \boldsymbol{f}$ and $b \nabla_{X} \nabla_{Y} \boldsymbol{f}=\nabla_{X} \nabla_{Y} \boldsymbol{f}$ for all $X, Y \in T_{p}$. Since the vectors $\nabla_{X} f, \nabla_{X} \nabla_{Y} f$ span the vector space $R^{m}$, we have $b=e$, the identity, and $c=0$, proving our assertion.

Proposition 2.2. Let $\boldsymbol{f}$ be an imbedaing of $M$ in $\boldsymbol{R}^{m}$.

(1) For all $A \in \mathfrak{e}(m), A f$ is a solution of the equation $\Phi_{* f}(\boldsymbol{u}) \quad 0$.

(2) If $\boldsymbol{f}$ satisfies condition $(C)$, then the map e $(m) \ni A \rightarrow A \boldsymbol{f} \in \Gamma(M, m)$ is injective.

This is clear from the invariance of $\Phi$ and Proposition 2.1.

Let $\rho(f)$ denote the dimension of the solution space of the equation $\Phi_{* f}(\boldsymbol{u})=0$, which is also the dimension of the solution space of the equation $L \varphi=0$ by Theorem 1.2. Then Proposition 2.2 implies

$$
\rho(\boldsymbol{f}) \geqq \operatorname{dim} E(m)=\frac{1}{2} m(m+1),
$$

provided that $f$ satisfies condition $(C)$. 
2.2. Upper semi-continuity of the dimension $\rho(f)$. Let us introduce the $C^{r}$-topology in the set $\Gamma(M, m)$. (Let $J^{r}$ be the vector bundle on $M$ consisting of all the $r$-jets of local differentiable maps of $M$ in $\boldsymbol{R}^{m}$. For $\boldsymbol{u} \in \Gamma(M, m)$, let $j_{p}^{r} \boldsymbol{u}$ denote the $r$-jet of $\boldsymbol{u}$ at $p \in M$. Then the assignment $p \rightarrow j_{p}^{r} \boldsymbol{u}$ gives a cross section $j^{r} \boldsymbol{u}$ of $J^{r}$, and the map $j^{r}: \Gamma(M, m)$ $\ni \boldsymbol{u} \rightarrow j^{r} \boldsymbol{u} \in \Gamma\left(J^{r}\right)$ is injective. This being said, the $C^{r}$-topology in $\Gamma(M, m)$ is the topology in $\Gamma(M, m)$ which is induced from the compact-open topology in $\Gamma\left(J^{r}\right)$ by the injective map $j^{r}$.) We denote by $\mathfrak{S}(M, m)_{C^{r}}$ the subset $\subseteq(M, m)$ of $\Gamma(M, m)$ equipped with the $C^{r}$-topology.

We shall prove the following

THEOREM 2.3 (cf. [4], Theorem 4). Let $f_{0}$ be an imbedding of $M$ in $\boldsymbol{R}^{m}$. We assume that $\boldsymbol{f}_{0}$ is elliptic and that $M$ is compact. Then there is a neighborhood $U_{1}\left(f_{0}\right)$ of $f_{0}$ in $\varsigma(M, m)_{C^{3}}$ such that $\rho(f) \leqq \rho\left(f_{0}\right)$ for every $f \in U_{1}\left(f_{0}\right)$.

In what follows, $\nabla^{f}, \tilde{N}^{f}, L^{f}$ with respectively mean the covariant differentiation $\nabla$, the bundle $\tilde{N}$, the operator $L$ which correspond to an imbedding $\boldsymbol{f} \in \mathfrak{S}(M, m)$.

The Riemannian metric $\nu=\Phi(f)$ differentiably depends on the 1 -jet $j^{1} \boldsymbol{f}$ of $\boldsymbol{f}$, that is, the components $g_{i j}$ of $\nu$ with respect to a coordinate system $x_{1}, \cdots, x_{n}$ of $M$ are differentiable functions of $j^{1} f: \nu=\sum_{i, j} g_{i j} d x_{i} d x_{j}$ and $g_{i j}=G_{i j} \circ j^{1} f$, where $G_{i j}$ are differentiable functions defined on an open set of $J^{1}$.

(2.1) It follows that the covariant differentiation $\nabla=\nabla^{f}$ differentiably depends on the 2-jet $j^{2} \boldsymbol{f}$ of $\boldsymbol{f}$, that is, the Riemann-Christoffel symbols $\Gamma_{j k}^{i}$ associated with $g_{i j}$ are differentiable functions of $j^{2} f$ in an analogous sense to the above.

(2.2) Hence the derivative $\nabla \nabla \boldsymbol{f}$ differentiably depends on $j^{2} \boldsymbol{f}$, that is, the components of $\nabla \nabla f$ with respect to the coordinate system $x_{1}, \cdots, x_{n}$ are differentiable functions of $j^{2} f$.

We choose once for all a subbundle $F$ of $S^{2} T^{*}$ complementary to $\tilde{N}^{f_{0}}$. By (2.2) we know that there is a neighborhood $V\left(f_{0}\right)$ of $f_{0}$ in $\mathfrak{S}(M, m)_{C^{2}}$ such that every $\boldsymbol{f} \in V\left(\boldsymbol{f}_{0}\right)$ is elliptic. If we choose $V\left(\boldsymbol{f}_{0}\right)$ sufficiently small, we also find that $S^{2} T^{*}=\tilde{N}^{f} \oplus F$ for every $f \in V\left(f_{0}\right)$. Hence the operator $L^{f}\left(f \in V\left(f_{0}\right)\right)$ may be regarded as an operator of $\Gamma\left(T^{*}\right)$ to $\Gamma(F)$. From (2.1) we infer that the operator $L^{f}$ differentiably depends on the 2-jet $j^{2} \boldsymbol{f}$ of $\boldsymbol{f}$. Namely let $x_{1}, \cdots, x_{n}$ be a coordinate system of 
$M$ at any point $p_{0}$ and let $\alpha_{1}, \cdots, \alpha_{l}$ be a moving frame of $F$ defined on a neighborhood of $p_{0}$. Then we have

$$
L^{f} \varphi=\sum_{\lambda}\left(\sum_{i, j} a_{i j}^{\lambda} \frac{\partial u_{i}}{\partial x_{j}}+\sum_{i} b_{i}^{i} u_{i}\right) \alpha_{\lambda}
$$

where $\varphi \in \Gamma\left(T^{*}\right)$ and $\varphi=\sum_{i} u_{i} d x_{i}$, and the components $a_{i j}^{\lambda}, b_{i}^{\lambda}$ are differentiable functions of $j^{2} \boldsymbol{f}$.

We are now able to prove Theorem 2.3 in a standard fashion by using some basic facts in the theory of elliptic operators. For completeness we shall accomplish the proof in Appendix.

\subsection{A rigidity theorem for elliptic imbeddings.}

THEOREM 2.4. Let $\boldsymbol{f}_{0}$ be an imbedding of $M$ in $\boldsymbol{R}^{m}$. We assume that $f_{0}$ is elliptic, $\rho\left(f_{0}\right)=\frac{1}{2} m(m+1)$ and that $M$ is compact. Then there is a neighborhood $U\left(f_{0}\right)$ of $f_{0}$ in $\mathfrak{E}(M, m)_{C^{3}}$ having the following property: If $\boldsymbol{f}, \boldsymbol{f}^{\prime} \in U\left(\boldsymbol{f}_{0}\right)$ and if $\Phi(\boldsymbol{f})=\Phi\left(\boldsymbol{f}^{\prime}\right)$, there is a unique Euclidean transformation $a$ of $\boldsymbol{R}^{m}$ such that $\boldsymbol{f}^{\prime}=a \boldsymbol{f}$.

Proof. For $\boldsymbol{f}, \boldsymbol{f}^{\prime} \in \mathfrak{F}(M, m)$, we put $\boldsymbol{u}=\boldsymbol{f}^{\prime}-\boldsymbol{f}$ and $\boldsymbol{h}=\boldsymbol{f}+\frac{1}{2} \boldsymbol{u}$. Then we can find a neighborhood $U\left(f_{0}\right)$ of $f_{0}$ in $\mathscr{E}(M, m)_{C^{3}}$ such that, for any $f, f^{\prime} \in U\left(f_{0}\right), \quad h$ is in $U_{1}\left(f_{0}\right)$, where $U_{1}\left(f_{0}\right)$ is a neighborhood of $f_{0}$ in $\mathfrak{S}(M, m)_{C^{3}}$ having the property in Theorem 2.3. Let $f, f^{\prime} \in U\left(f_{0}\right)$ be such that $\Phi(\boldsymbol{f})=\Phi\left(\boldsymbol{f}^{\prime}\right)$. This clearly gives $\Phi_{* \boldsymbol{h}}(\boldsymbol{u})=2\langle d \boldsymbol{h}, d \boldsymbol{u}\rangle=0$. Since $\boldsymbol{h} \in$ $U_{1}\left(f_{0}\right)$, we have

$$
\frac{1}{2} m(m+1) \leqq \rho(\boldsymbol{h}) \leqq \rho\left(\boldsymbol{f}_{0}\right)=\frac{1}{2} m(m+1),
$$

whence $\rho(\boldsymbol{h})=\frac{1}{2} m(m+1)$. From these facts and Proposition 2.2 we see that there are a unique $B \in \mathfrak{D}(m)$ and a unique $\boldsymbol{c} \in \boldsymbol{R}^{m}$ such that

$$
\boldsymbol{u}=B \boldsymbol{h}+\boldsymbol{c}=B\left(\boldsymbol{f}+\frac{1}{2} \boldsymbol{u}\right)+\boldsymbol{c} .
$$

We have $\operatorname{det}\left(1-\frac{1}{2} B\right) \neq 0$, because $B$ is a skew-symmetrix matrix. Therefore it follows that

$$
\boldsymbol{u}=\left(1-\frac{1}{2} B\right)^{-1} B \boldsymbol{f}+\left(1-\frac{1}{2} B\right)^{-1} \boldsymbol{c}
$$

and hence

$$
\boldsymbol{f}^{\prime}=\left(1+\left(1-\frac{1}{2} B\right)^{-1} B\right) \boldsymbol{f}+\left(1-\frac{1}{2} B\right)^{-1} \boldsymbol{c} .
$$

An easy calculation shows that $1+\left(1-\frac{1}{2} B\right)^{-1} B$ is an orthogonal matrix, 
proving the existence part of Theorem 2.4. The uniqueness part follows from Proposition 2.1.

2.4. Remarks on the operator $L$. Let $\boldsymbol{f}$ be an imbedding of $M$ in $\boldsymbol{R}^{m}$ satisfying condition $(C)$. If we put $n=\operatorname{dim} M$, then we have $m=n+$ $\operatorname{dim} N$ and $\operatorname{dim} N=\operatorname{dim} \tilde{N} \leqq \operatorname{dim} S^{2} T^{*}=\frac{1}{2} n(n+1)$, where $N$ (resp. $\left.\tilde{N}\right)$ is the normal bundle (resp. the bundle of second fundamental forms) of $f$. Hence the dimensions $n$ and $m$ must satisfy the inequalities:

$$
n \leqq m \leqq \frac{1}{2} n(n+3) \text {. }
$$

We shall now explain (without proof) how the properties of the operator $L$ associated with $\boldsymbol{f}$ depend on the dimensions $n$ and $m$ and how the operator is connected with the imbedding problem for Riemannian manifolds.

a) The operator $L$ is over-determined when $n \leqq m<\frac{1}{2} n(n+1)$.

b) Proposition 2.5. In order that the operator $L$ is elliptic, it is necessary that ${ }^{3)} n \leqq m<\frac{1}{2} n(n+1)$ or $n=2$ and $m=3$.

c) The fibre $\tilde{N}_{p}$ of $\tilde{N}$ at $p \in M$, being a subspace of $S^{2} T_{p}^{*}$, may be identified with a vector space of symmetric endomorphisms of the Euclidean vector space $T_{p}$ (The inner product in $T_{p}$ is defined by the Riemannian metric $\nu=\Phi(f)$.). This being said, we define a subspace $\mathfrak{g}_{p}$ of $\operatorname{Hom}\left(T_{p}, T_{p}\right)$ by $\mathfrak{g}_{p}=\mathfrak{o}\left(T_{p}\right)+\tilde{N}_{p}$, where $\mathfrak{o}\left(T_{p}\right)$ is the vector space of skew-symmetric endomorphisms of $T_{p}$. We note that the vector bundle $\mathfrak{g}=\bigcup_{p} \mathfrak{g}_{p}$ may be regarded as the $\operatorname{symbol}^{4)}$ of the equation $L \varphi=0$. Let $\mathrm{g}_{p}^{(i)}$ be the $i$-th prolongation ${ }^{5)}$ of the subspace $\mathfrak{g}_{p}$ of Hom $\left(T_{p}, T_{p}\right)$. Then we have $\operatorname{dim} \mathfrak{g}_{p}^{(1)}=n \operatorname{dim} \tilde{N}$.

We say that the imbedding $\boldsymbol{f}$ is involutive if $\mathfrak{g}_{p}$ is involutive $\mathrm{e}^{6)}$ at every $p \in M$. In order that $\boldsymbol{f}$ is involutive, it is necessary that $\frac{1}{2} n(n+1)$ $\leqq m \leqq \frac{1}{2} n(n+3)$.

Proposition 2.6. The imbedding $\boldsymbol{f}$ is involutive if and only if the equation $L \varphi=0$ is involutive.

THEOREM 2.7. If $\boldsymbol{f}$ is involutive, then the equation $\Phi_{* \boldsymbol{f}}(\boldsymbol{u})=\alpha$ has a local solution for any given local cross section $\alpha$ of $S^{2} T^{*}$, where everything should be considered in the real analytic category.

3) It is easy to show that $n \leqq m<\frac{1}{2} n(n+1)$ or $n$ is even and $m=\frac{1}{2} n(n+1)$. The second case occurs only when $n=2$, which follows immediately from [1], Theorem 1 .

4) See [8].

5) and ${ }^{6)}$ See [9]. 
The É. Cartan's result [2] indicates that every Riemannian manifold $M$ of dimension $n$ can be locally isometrically imbedded in the Euclidean space of dimension $\frac{1}{2} n(n+1)$ by an involutive imbedding, where as above everything should be considered in the real analytic category.

d) The case where $m=\frac{1}{2} n(n+3)$. Since $\tilde{N}=S^{2} T^{*}$, the operator $L$ is reduced to 0 and hence the equation $\Phi_{* f}(\boldsymbol{u})=\alpha$ has a global solution for any given $\alpha \in \Gamma\left(S^{2} T^{*}\right)$, which is a basic fact in the theory of Nash. [6]. An example of such an imbedding is the canonical isometric imbedding of the real projective space $P^{n}(\boldsymbol{R})$ in the space of symmetric matrices of degree $n+1$ with vanishing trace (cf. 3.1, Example.)

e) The case where $n=2$ and $m=3$. The imbedding $f$ is always involutive, and it is elliptic if and only if the second fundamental form corresponding to any normal vector $(\neq 0)$ is definite. Consider the case where $M=S^{2}$, the unit sphere in $R^{3}$. Then $f$ is elliptic if and only if the image $f\left(S^{2}\right)$ of $S^{2}$ by $\boldsymbol{f}$ is an ovaloid in $\boldsymbol{R}^{3}$, and when $\boldsymbol{f}$ is elliptic, we know the following facts: $1^{0} . \rho(f)=\frac{1}{2} m(m+1)=6,2^{0}$. the equation $\Phi_{* f}(\boldsymbol{u})=\alpha$ has a global solution for any given $\alpha \in \Gamma\left(S^{2} T^{*}\right)$. These facts play an important role in the solution [7] of the Weyl problem.

f) The case where $n \geqq 3$ and $m=n+1$. Consider the case where the rank of the second fundamental form corresponding to any normal vector $(\neq 0)$ is at least 3 . Then the imbedding $f$ is elliptic, and we can prove the following facts: $1^{0}$. The equation $L \varphi=0$ is of finite type or more precisely, $\operatorname{dim} \mathfrak{g}_{p}=\frac{1}{2} n(n-1)+1, \operatorname{dim} \mathfrak{g}_{p}^{(1)}=n$ and $\mathfrak{g}_{p}^{(2)}=0,2^{0}$. the equation is formally integrable ${ }^{7)}, 3^{0} . \quad \rho(f)=\frac{1}{2} m(m+1)$ without compactness assumption.

\section{§3. Rigidity for some classes of elliptic imbeddings}

3.1. The canonical isometric imbedding of a compact hermitian symmetric space. Let $M$ be a global hermitian symmetric space. (For the theory of hermitian symmetric spaces, we refer to [3].) Let $I$ and $\nu$ be the almost complex structure and the Riemannian metric respectively on $M$. Let $G$ be the largest connected group of automorphisms (holomorphic isometries) of $M$. The group $G$ transitively acts on $M$ and hence the space $M$ may be expressed as the homogeneous space $G / H$, where $H$ is the isotropy group of $G$ at a fixed point 0 of $M$. In the following we assume that $G$ is compact and semi-simple.

7) See [8]. 
Let $g$ be the Lie algebra of $G$ and $B$ its Killing form. Note that $B$ is negative definite. Let $\mathfrak{h}$ be the Lie algebra of $H$ and $\mathfrak{n}$ the orthogonal complement of $\mathfrak{h}$ in $\mathfrak{g}$ with respect to the Killing form $B$. We have $[\mathfrak{h}, \mathfrak{m}] \subset \mathfrak{m}$ and $[\mathfrak{m}, \mathfrak{m}] \subset \mathfrak{h}$. Let $\pi$ be the projection of $G$ onto $M$. Then the linear map $\mathfrak{m} \ni X \rightarrow \pi_{*} X_{e} \in T_{0}=T(M)_{0}$ is an isomorphism, by which we shall identify the two vector spaces $m$ and $T_{0}$. This being said, we make the second assumption that the Riemannian metric $\nu$ is induced from the Killing form $B$, that is,

$$
\nu(X, Y)=-B(X, Y)
$$

for all $X, Y \in T_{0}$.

It is well known that there is a unique element $Z_{0}$ in the centre of $\mathfrak{h}$ such that $I X=\left[Z_{0}, X\right]$ for all $X \in T_{0}$ and such that $H$ is the centralizer of $Z_{0}$ in $G$, i.e., $H=\left\{a \in G \mid \operatorname{ad} a Z_{0}=Z_{0}\right\}$. (See [3], Theorem 9.6.) By the second property of $Z_{0}$, we see that the map $G \ni a \rightarrow \operatorname{ad} a Z_{0} \in g$ induces a map $f$ of $M$ to g. Furthermore we see that $f$ is an imbedding and that it is equivariant, i.e.,

$$
\boldsymbol{f}(a p)=\operatorname{ad} a \boldsymbol{f}(p)
$$

for all $a \in G$ and $p \in M$.

Let us now define an inner product $\langle$,$\rangle in \mathfrak{g}$ as follows:

$$
\langle X, Y\rangle=-B(X, Y)
$$

for all $X, Y \in g$. Thus if we put $m=\operatorname{dim} g, g$ may be regarded as the $m$-dimensional Euclidean space $\boldsymbol{R}^{m}$ with respect to a fixed orthonormal base $X_{1}, \cdots, X_{m}$ of $\mathrm{g}$. Note that ad $\alpha: \mathfrak{g} \rightarrow \mathfrak{g}$ is an orthogonal transformation for all $a \in G$. Let $\nabla$ be the covariant differentiation associated with the Riemannian metric $\nu$ and $R$ its curvature.

Proposition 3.1. (1) $\boldsymbol{f}$ is an isometric imbedding of the hermitian symmetric space $M$ in the Euclidean space $\mathfrak{g}=\boldsymbol{R}^{m}$ ([5]).

(2) $f$ satisfies condition (C).

(3) $\nabla_{Z} \nabla_{X} \nabla_{Y} f=(R(I X, Y) I Z) f$

for all $X, Y, Z \in T_{p}$ and $p \in M$.

First we shall prove the following.

LEMMA 3.2. For all $X, Y \in T_{0}=\mathfrak{m}$, we have: 
(1) $X \boldsymbol{f}=-I X$.

(2) $\nabla_{X} \nabla_{Y} f=[I X, Y]=-[X, I Y]$.

Proof. If we put $u(t)=\pi(\exp t X)$, we have

$$
f(u(t))=\operatorname{ad}(\exp t X) Z_{0}=\sum_{k} \frac{t^{k}}{k !}(\operatorname{ad} X)^{k} Z_{0} .
$$

We have $(d u / d t)(0)=\pi_{*} X=X$ and hence

$$
X \boldsymbol{f}=\operatorname{ad} X Z_{0}=\left[X, Z_{0}\right]=-I X,
$$

proving (1). Let $\tilde{X}$ be the vector field on $M$ induced by the 1-parameter group of transformations: $\boldsymbol{R} \times M \ni(t, p) \rightarrow(\exp t X) p \in M$. Then $\left(d^{2} \boldsymbol{f} \circ u /\right.$ $\left.d t^{2}\right)(0)=X \tilde{X} \boldsymbol{f}$ and hence

$$
X \tilde{X} \boldsymbol{f}=(\operatorname{ad} X)^{2} Z_{0}=\left[X,\left[X, Z_{0}\right]\right]=[I X, X] .
$$

On the other hand

$$
X \tilde{X} \boldsymbol{f}=\nabla_{X} \nabla_{X} \boldsymbol{f}+\left(\nabla_{X} \tilde{X}\right) \boldsymbol{f} .
$$

$u(t)$ is an integral curve of $\tilde{X}$ and at the same time a geodesic, whence $\nabla_{X} \tilde{X}=0$. Therefore $\nabla_{X} \nabla_{X} f=[I X, X]$. Since $\nabla_{X} \nabla_{Y} f=\nabla_{Y} \nabla_{X} f$ and since $[I X, Y]+[X, I Y]=\left[Z_{0},[X, Y]\right]=0$, it follows that $\nabla_{X} \nabla_{Y} f=[I X, Y]$, proving (2).

Proof of Proposition 3.1. By Lemma 3.2, (1) we have $\langle X f, Y \boldsymbol{f}\rangle=$ $-B(I X, I Y)=-B(X, Y)=\nu(X, Y)$ for all $X, Y \in T_{0}$. Since $f$ is equivariant, it follows that $\langle X f, Y \boldsymbol{f}\rangle=\nu(X, Y)$ for all $X, Y \in T_{p}$ and $p \in M$, proving (1). We have $\mathfrak{h}=[\mathfrak{m}, \mathfrak{m}]$. Hence (2) follows from Lemma 3.2, (2) and the equivariance of $f$. By Lemma 3.2 and the equivariance of $f$, we have

$$
\nabla_{X} \nabla_{Y} f=\left[\nabla_{I X} f, \nabla_{Y} f\right]
$$

for all $X, Y \in T_{p}$ and $p \in M$. Therefore by using $\nabla I=0$, we have, for all $Z \in T_{p}$,

$$
\begin{aligned}
\nabla_{Z} \nabla_{X} \nabla_{Y} f & =\left[\nabla_{Z} \nabla_{I X} f, \nabla_{Y} f\right]+\left[\nabla_{I X} f, \nabla_{Z} \nabla_{Y} f\right] \\
& =\left[\left[\nabla_{I Z} f, \nabla_{I X} f\right], \nabla_{Y} f\right]+\left[\nabla_{I X} f,\left[\nabla_{I Z} f, \nabla_{Y} f\right]\right] \\
& =\left[\nabla_{I Z} f,\left[\nabla_{I X} f, \nabla_{Y} f\right]\right] .
\end{aligned}
$$

Considering the case where $p=0$ and using Lemma 3.2, (1), we have 


$$
\nabla_{Z} \nabla_{X} \nabla_{Y} f=-[Z,[X, I Y]]
$$

for all $X, Y, Z \in T_{0}$. Since

$$
R(I X, Y) I Z=-[[I X, Y], I Z],
$$

it follows that

$$
\nabla_{Z} \nabla_{X} \nabla_{Y} \boldsymbol{f}=-[[I X, Y], Z]=(R(I X, Y) I Z) \boldsymbol{f} .
$$

Now (3) follows from this equality and the equivariance of $\boldsymbol{f}$.

We denote by $E$ the subbundle of $S^{2} T^{*}$ which consists of all the hermitian forms $\left(\in S^{2} T^{*}\right)$ with respect to the almost complex structure I. As is easily observed, $E$ is elliptic.

Proposition 3.3. The bundle $\tilde{N}$ of second fundamental forms of $\boldsymbol{f}$ is a subbundle of the bundle $E$ of hermitian forms. In particular the imbedding $\boldsymbol{f}$ is elliptic.

Proof. By Lemma 3.2, (2) and the equivariance of $f$, we have

$$
\nabla_{X} \nabla_{Y} f=\nabla_{I X} \nabla_{I Y} f
$$

for all $X, Y \in T_{p}$. Proposition 3.3 is immediate from this fact.

ExAmple. Let us consider the case where the hermitian symmetric space $M=G / H$ is the $n$-dimensional complex projective space $P^{n}(C)$. We have

$$
G=U(n+1) / C \text { and } H=U(1) \times U(n) / C,
$$

where $C$ is the centre of $U(n+1)$ and the isotropy group $H$ is considered at the point $0=(1,0, \cdots, 0)$. Hence $\mathfrak{g} \cong \mathfrak{I} \mathfrak{u}(n+1)$ and $\operatorname{dim} \mathfrak{g}=$ $n^{2}+2 n$. Let $z_{0}, \cdots, z_{n}$ be the homogeneous coordinates of $P^{n}(C)$. Then the imbedding $\boldsymbol{f}=\boldsymbol{f}^{(n)}$ is given by

$$
f^{(n)}\left(\left(z_{0}, \cdots, z_{n}\right)\right)=\sqrt{-1}\left(\frac{\delta_{i j}}{n+1}-\frac{z_{i} \bar{z}_{j}}{|z|^{2}}\right) \quad(\in \mathfrak{S} \mathfrak{u}(n+1)),
$$

where $|z|^{2}=\sum_{k}\left|z_{k}\right|^{2}$. Finally we note that the two bundles $\tilde{N}$ and $E$ just coincide.

3.2. A rigidity theorem associated with the isometric imbedding $f: M \rightarrow g$. In the next paragraph we shall prove the following

THEOREM 3.4. Let $\boldsymbol{f}$ be the isometric imbedding of the compact 
hermitian symmetric space $M$ in the Euclidean space $\mathfrak{g}=\boldsymbol{R}^{m}$ defined in 3.1. Then we have $\rho(\boldsymbol{f})=\frac{1}{2} m(m+1)$.

By Proposition 3.3, Theorems 2.4 and 3.4 we have the following

THEOREM 3.5. Let $\boldsymbol{f}$ be as in Theorem 3.4. Then there is a neighborhood $U(\boldsymbol{f})$ of $\boldsymbol{f}$ in $\left[(E, m)_{C^{3}}\right.$ having the following property: If $\boldsymbol{f}^{\prime}, \boldsymbol{f}^{\prime \prime}$ $\in U(f)$ and if $\Phi\left(f^{\prime}\right)=\Phi\left(f^{\prime \prime}\right)$, there is a unique Euclidean transformation $a$ of g such that $\boldsymbol{f}^{\prime \prime}=a f^{\prime}$.

Remark 1. Consider the isometric imbedding $f^{(1)}$ of $P^{1}(C)$ in $\mathfrak{s} \mathfrak{u}(2)$ $=\boldsymbol{R}^{3}$. (See 3.1, Example.) $P^{1}(\boldsymbol{C})$ is isomorphic with the sphere $S^{2}(\sqrt{2})$ of radius $\sqrt{2}$ in $R^{3}$ as Riemannian manifolds. Therefore Theorem 3.5 is a partial generalization of the theorem of Cohn-Vossen.

Remark 2. It is well known that the sphere $S^{2}(\sqrt{2})$ in $\boldsymbol{R}^{3}$ or the isometric imbedding $\boldsymbol{f}^{(1)}$ is locally deformable. (For example, see [2].) Therefore the isometric imbedding $f$ of the hermitian symmetric space $M$ in $g$ is locally deformable, provided that $P^{1}(C)$ appears in the de Rham decomposition of $M$. We shall see in the proof of Theorem 3.4 that the equation $L \varphi=0$ associated with the isometric imbedding $\boldsymbol{f}^{(n)}$ of $P^{n}(C)$ in $\mathfrak{Z} \mathfrak{u}(n+1)$ is of infinite type, which suggests that $\boldsymbol{f}^{(n)}$ is locally deformable. However the problem to examine it seems to be rather complicated and difficult.

3.3. Proof of Theorem 3.4. The proof is devided into several steps.

I. $M$ is a Kahlerian manifold: $\nabla I=0$ and $M$ is also a symmetric space: $\nabla R=0$. Since the Riemannian metric $\nu$ is induced from the Killing form $B$ of $\mathfrak{g}$, we know that $M$ is an Einstein space ([3], Proposition 9.7):

$$
\sum_{i} R\left(X, e_{i}\right) e_{i}=-\frac{1}{2} \sum_{i} R\left(I e_{i}, e_{i}\right) I X=\frac{1}{2} X .
$$

(In the following $X, Y, Z$ will denote any vectors at any point $p \in M$ and $e_{1}, \cdots, e_{2 n}$ any orthogonal frame at the point $p$, where $n=\operatorname{dim}_{C} M$.)

For $\alpha, \beta \in \Gamma\left(\otimes^{p} T^{*}\right)$ we define a function $\langle\alpha, \beta\rangle$ by

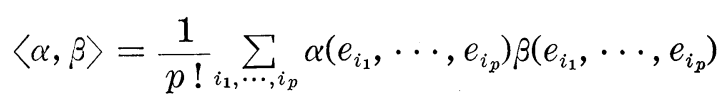

and put 


$$
(\alpha, \beta)=\int_{M}\langle\alpha, \beta\rangle d v,
$$

the inner product of $\alpha$ and $\beta$, where $d v$ is the volume element of the oriented Riemannian manifold $M$.

Let $\delta$ be the adjoint operator of the exterior differentiation $d$ of $\Gamma\left(\Lambda^{p} T^{*}\right)$ to $\Gamma\left(\Lambda^{p+1} T^{*}\right)$ with respect to the inner products (, ). Then the operator $\Delta=\delta d+d \delta$ is the so called Laplacian. In terms of $\nabla$, we have:

$$
\begin{aligned}
& (d \varphi)\left(X_{1}, \cdots, X_{p+1}\right)=\sum_{i}(-1)^{i-1}\left(\nabla_{X_{i}} \varphi\right)\left(X_{1}, \cdots, \hat{X}_{i}, \cdots, X_{p+1}\right), \\
& (\delta \varphi)\left(X_{1}, \cdots, X_{p}\right)=-\sum_{i}\left(\nabla_{e_{i}} \varphi\right)\left(e_{i}, X_{1}, \cdots, X_{p}\right) .
\end{aligned}
$$

We have

$$
\Delta f=-\sum_{i} \nabla_{e_{i}} \nabla_{e_{i}} f
$$

for a function $f$ and

$$
\Delta \varphi=-\sum_{i} \nabla_{e_{i}} \nabla_{e_{i}} \varphi+\frac{1}{2} \varphi
$$

for a 1 -form $\varphi$.

II. Let $E^{\prime}$ be the subbundle of $S^{2} T^{*}$ which consists of all the antihermitian forms $\left(\in S^{2} T^{*}\right)$ with respect to $I: S^{2} T^{*}=E \oplus E^{\prime} . \quad\left(\alpha \in S^{2} T^{*}\right.$ is anti-hermitian if $\alpha(I X, I Y)=-\alpha(X, Y)$.) We define a differential operator $L_{0}$ of $\Gamma\left(T^{*}\right)$ to $\Gamma\left(E^{\prime}\right)$ by

$$
\left(L_{0} \varphi\right)(X, Y)=\frac{1}{2}((D \varphi)(X, Y)-(D \varphi)(I X, I Y))
$$

for all $\varphi \in \Gamma\left(T^{*}\right)$, i.e., $L_{0} \varphi$ is the anti-hermitian part of $D \varphi . \tilde{N}$ is a subbundle of $E$ by Proposition 3.3 and hence a solution of the equation $L \varphi=0$ is necessarily a solution of the equation $L_{0} \varphi=0$.

Remark. Let $\varphi$ be a local 1 -form on $M$ and $X$ the vector field dual to $\varphi$ with respect to $\nu$. The fact that $\varphi$ is a solution of the equation $L_{0} \varphi=0$ means that $D \varphi=L_{X} \nu, L_{X}$ being the Lie derivation, is hermitian. Hence if $X$ is an analytic vector field, then $\varphi$ is a solution of the equation $L_{0} \varphi=0$. It follows that the equation $L_{0} \varphi=0$ is of infinite type. In particular consider the case where $M=P^{n}(C)$. Then $\tilde{N}=E$ and hence $L$ may be identified with $L_{0}$. Consequently the equation $L \varphi=0$ is of infinite type.

Let $L_{0}^{*}$ be the adjoint operator of $L_{0}$ with respect to the inner products (, ). In terms of $\nabla, L_{0}^{*}$ may be expressed as follows: 


$$
\left(L_{0}^{*} \alpha\right)(X)=-\sum_{i}\left(\nabla_{e_{i}} \alpha\right)\left(e_{i}, X\right)
$$

for all $\alpha \in \Gamma\left(E^{\prime}\right)$. Indeed we have, for all $\varphi \in \Gamma\left(T^{*}\right)$,

$$
\begin{aligned}
\left\langle L_{0} \varphi, \alpha\right\rangle & =\sum_{i, j}\left(\nabla_{e_{i}} \varphi\right)\left(e_{j}\right) \alpha\left(e_{i}, e_{j}\right) \\
& =\sum_{i}\left(\nabla_{e_{i}} \beta\right)\left(e_{i}\right)-\sum_{i, j} \varphi\left(e_{j}\right)\left(\nabla_{e_{i}} \alpha\right)\left(e_{i}, e_{j}\right),
\end{aligned}
$$

where $\beta$ is the 1 -form defined by $\beta(X)=\sum_{j} \varphi\left(e_{j}\right) \alpha\left(X, e_{j}\right)$. Since the integral of $\delta \beta \cdot d v$ over $M$ is 0 , we obtain the desired formula.

For $\varphi \in \Gamma\left(T^{*}\right)$ we define $\varphi I \in \Gamma\left(T^{*}\right)$ by $(\varphi I)(X)=\varphi(I X)$.

LEMMA 3.6 .

$$
2 L_{0}^{*} L_{0} \varphi=\Delta \varphi+d \delta \varphi-(d \delta(\varphi I)) I-2 \varphi .
$$

Proof. We have:

$$
\begin{aligned}
\sum_{i}\left(\nabla_{e_{i}} D \varphi\right)\left(e_{i}, X\right) & =\sum_{i}\left(\nabla_{e_{i}} \nabla_{e_{i}} \varphi\right)(X)+\sum_{i}\left(\nabla_{e_{i}} \nabla_{X} \varphi\right)\left(e_{i}\right), \\
\sum_{i}\left(\nabla_{e_{i}} \nabla_{X} \varphi\right)\left(e_{i}\right)= & \sum_{i}\left(\nabla_{X} \nabla_{e_{i}} \varphi\right)\left(e_{i}\right)-\sum_{i} \varphi\left(R\left(e_{i}, X\right) e_{i}\right) \\
= & \left(-d \delta \varphi+\frac{1}{2} \varphi\right)(X) .
\end{aligned}
$$

\section{Hence}

$$
\sum_{i}\left(\nabla_{e_{i}} D \varphi\right)\left(e_{i}, X\right)=(-\Delta \varphi-d \delta \varphi+\varphi)(X)
$$

We have:

$$
\begin{aligned}
\sum_{i}\left(\nabla_{e_{i}} D \varphi\right)\left(I e_{i}, I X\right) & =\sum_{i}\left(\nabla_{e_{i}} \nabla_{I e_{i}} \varphi\right)(I X)+\sum_{i}\left(\nabla_{e_{i}} \nabla_{I X} \varphi\right)\left(I e_{i}\right), \\
\sum_{i}\left(\nabla_{e_{i}} \nabla_{I e_{i}} \varphi\right)(I X) & =-\sum_{i}\left(\nabla_{I e_{i}} \nabla_{e_{i}} \varphi\right)(I X) \\
= & -\sum_{i}\left(\nabla_{e_{i}} \nabla_{I e_{i}} \varphi\right)(I X)+\sum_{i} \varphi\left(R\left(I e_{i}, e_{i}\right) I X\right), \\
\sum_{i}\left(\nabla_{e_{i}} \nabla_{I X} \varphi\right)\left(I e_{i}\right) & =\left(-(d \delta(\varphi I)) I-\frac{1}{2} \varphi\right)(X) .
\end{aligned}
$$

Hence $\sum_{i}\left(\nabla_{e_{i}} \nabla_{I_{i}} \varphi\right)(I X)=-\frac{1}{2} \varphi(X)$ and

$$
\sum_{i}\left(\nabla_{e_{i}} D \varphi\right)\left(I e_{i}, I X\right)=(-(d \delta(\varphi I)) I-\varphi)(X) .
$$

Lemma 3.6 is immediate from (3.1) and (3.2).

III. By Lemma 3.6, every solution of the equation $L_{0} \varphi=0$ is a solution of the equation: 


$$
\Delta \varphi=-d \delta \varphi+(d \delta(\varphi I)) I+2 \varphi
$$

and the converse is also true. Let us now consider the following equation:

$$
\Delta f=f,
$$

$f$ being functions.

LEMMA 3.7. (1) If $f$ is a solution of equation (3.4), then $d f$ is a solution of equation (3.3).

(2) Every solution $\varphi$ of equation (3.3) can be uniquely written as

$$
\varphi=d f+\varphi_{1}
$$

where $f$ is a solution of equation (3.4) and $\varphi_{1}$ is a solution of equation (3.3) combined with the equation $\delta \varphi=0$.

Proof. This lemma is easy from the following facts: $d \Delta=\Delta d$, $\delta \Delta=\Delta \delta$ and $\delta((d f) I)=0$ for any function $f$.

A 1 -form $\varphi$ on $M$ is called a Killing form if the vector field $X$ dual to $\varphi$ with respect to $\nu$ is a Killing vector field, i.e., $L_{X} \nu=D \varphi=0$.

LEMMA 3.8. The solutions $f$ of equation (3.4) are in a one-to-one correspondence with the Killing forms $\varphi$ and the correspondence $f \rightarrow \varphi$ is given by the relation $\varphi=(d f) I$. In particular the solutions of equation (3.4) form a vector space of dimension $m(=\operatorname{dim} \mathfrak{g})$.

This fact is well known. (For example, see [12], Chapter IV.) The next lemma is also known.

LEMMA 3.9. If we put $\boldsymbol{f}=\left(f_{1}, \cdots, f_{m}\right)$, i.e., $\boldsymbol{f}=\sum_{i} f_{i} X_{i}$, then the functions $f_{1}, \cdots, f_{m}$ form a base of the solution space of equation (3.4).

Proof. Let $e_{1}, \cdots, e_{2 n}$ be an orthonormal base of $m=T_{0}$. Then we have

$$
-\sum_{i}\left[\left[I e_{i}, e_{i}\right], X\right]=\sum_{i} R\left(I e_{i}, e_{i}\right) X=I X=\left[Z_{0}, X\right]
$$

for all $X \in T_{0}$, whence $\sum_{i}\left[I e_{i}, e_{i}\right]=-Z_{0}$. Therefore by Lemma 3.2, (2), we have

$$
\sum_{i} \nabla_{e_{i}} \nabla_{e_{i}} f=\sum_{i}\left[I e_{i}, e_{i}\right]=-Z_{0}=-f(0)
$$


This together with the equivariance of $\boldsymbol{f}$ yields $\Delta \boldsymbol{f}=\boldsymbol{f}$. Hence $f_{1}, \cdots, f_{m}$ are solutions of equation (3.4). Lemma 3.9 now follows from Lemma 3.8.

LEMMA 3.10. If $f$ is a solution of equation (3.4), then $d f$ is a solution of the equation $L \varphi=0$.

Proof. By Lemma 3.9, it is sufficient to prove this for $f=f_{i}$. We have $D d f_{i}=2 \nabla \nabla f_{i}$, and $\nabla \nabla f_{i}=\left\langle X_{i}, \nabla \nabla \boldsymbol{f}\right\rangle$ is a cross section of $\tilde{N}$. Hence we obtain $L d f_{i}=0$.

Remark. It can be proved that the dimension of the solution space of the equation $L_{0} \varphi=0$ is equal to $l+2 m$, where $l$ is the dimension of the solution space of the equation $\Delta \varphi=2 \varphi, \varphi$ being 1 -forms. Consequently in the case where $M=P^{n}(C)$, our problem is reduced to the problem to find the dimension $l$.

IV. Let $\boldsymbol{u}$ be a solution of the equation $\Phi_{* f}(\boldsymbol{u})=0$. By Theorem 1.2 there corresponds to $\boldsymbol{u}$ a solution $\varphi$ of the equation $L \varphi=0$. Since $\varphi=\langle\boldsymbol{u}, d \boldsymbol{f}\rangle$, we have

$$
\left\langle\boldsymbol{u}, \nabla_{Y} \boldsymbol{f}\right\rangle=\varphi(Y)
$$

and hence

$$
\left\langle\nabla_{X} \boldsymbol{u}, \nabla_{Y} \boldsymbol{f}\right\rangle+\left\langle\boldsymbol{u}, \nabla_{X} \nabla_{Y} \boldsymbol{f}\right\rangle=\left(\nabla_{X} \varphi\right)(Y) \text {. }
$$

We have $\Phi_{* f}(\boldsymbol{u})=2\langle d \boldsymbol{f}, d \boldsymbol{u}\rangle=0$, meaning that $\left\langle\nabla_{X} \boldsymbol{u}, \nabla_{Y} \boldsymbol{f}\right\rangle$ is skew-symmetric with respect to $X$ and $Y$. Furthermore $\left\langle\boldsymbol{u}, \nabla_{X} \nabla_{Y} \boldsymbol{f}\right\rangle$ is symmetric with respect to $X$ and $Y$. Therefore we obtain:

$$
\begin{aligned}
& \left\langle\boldsymbol{u}, \nabla_{X} \nabla_{Y} \boldsymbol{f}\right\rangle=\frac{1}{2}(D \varphi)(X, Y), \\
& \left\langle\nabla_{X} \boldsymbol{u}, \nabla_{Y} \boldsymbol{f}\right\rangle=\frac{1}{2}(d \varphi)(X, Y) .
\end{aligned}
$$

LEMMA 3.11. (1) $\left\langle\Delta \boldsymbol{u}-\boldsymbol{u}, \nabla_{X} \boldsymbol{f}\right\rangle=-(d \delta \varphi)(X)$.

(2) $\left\langle\Delta \boldsymbol{u}-\boldsymbol{u}, \nabla_{X} \nabla_{Y} \boldsymbol{f}\right\rangle=\left(\frac{1}{2} D \Delta \varphi-D \varphi\right)(X, Y)+\sum_{i}\left(L_{0} \varphi\right)\left(R\left(e_{i}, Y\right) X, e_{i}\right)$.

Proof. By (3.5) and (3.6) we have

$$
\begin{aligned}
& \sum_{i}\left\langle\nabla_{e_{i}} \boldsymbol{u}, \nabla_{e_{i}} \nabla_{X} \boldsymbol{f}\right\rangle+\sum_{i}\left\langle\boldsymbol{u}, \nabla_{e_{i}} \nabla_{e_{i}} \nabla_{X} \boldsymbol{f}\right\rangle=\frac{1}{2} \sum_{i}\left(\nabla_{e_{i}} D \varphi\right)\left(e_{i}, X\right), \\
& \sum_{i}\left\langle\nabla_{e_{i}} \nabla_{e_{i}} \boldsymbol{u}, \nabla_{X} \boldsymbol{f}\right\rangle+\sum_{i}\left\langle\nabla_{e_{i}} \boldsymbol{u}, \nabla_{e_{i}} \nabla_{X} \boldsymbol{f}\right\rangle=\frac{1}{2} \sum_{i}\left(\nabla_{e_{i}} d \varphi\right)\left(e_{i}, X\right) .
\end{aligned}
$$

By Proposition 3.1, (3) we have 


$$
\begin{aligned}
\sum_{i}\left\langle\boldsymbol{u}, \nabla_{e_{i}} \nabla_{e_{i}} \nabla_{X} \boldsymbol{f}\right\rangle= & \sum_{\imath}\left\langle\boldsymbol{u},\left(R\left(I e_{i}, X\right) I e_{i}\right) \boldsymbol{f}\right\rangle \\
& -\frac{1}{2}\langle\boldsymbol{u}, X \boldsymbol{f}\rangle=-\frac{1}{2} \varphi(X) .
\end{aligned}
$$

Hence we obtain

$$
\begin{aligned}
\left\langle\Delta \boldsymbol{u}-\boldsymbol{u}, \nabla_{X} \boldsymbol{f}\right\rangle= & \frac{1}{2}(-\Delta \varphi-d \delta \varphi+\varphi)(X) \\
& +\frac{1}{2}(\delta d \varphi)(X)-\frac{1}{2} \varphi(X) \\
= & -(d \delta \varphi)(X),
\end{aligned}
$$

proving (1).

By (3.5) and Proposition 3.1, (3) we have

$$
\left\langle\nabla_{Z} \boldsymbol{u}, \nabla_{X} \nabla_{Y} \boldsymbol{f}\right\rangle+\varphi(R(I X, Y) I Z)=\frac{1}{2}\left(\nabla_{Z} D \varphi\right)(X, Y) .
$$

It follows that

$$
\begin{aligned}
& \sum_{i}\left\langle\nabla_{e_{i}} \nabla_{e_{i}} \boldsymbol{u}, \nabla_{X} \nabla_{Y} \boldsymbol{f}\right\rangle+\sum_{i}\left\langle\nabla_{e_{i}} \boldsymbol{u}, \nabla_{e_{i}} \nabla_{X} \nabla_{Y} \boldsymbol{f}\right\rangle \\
& \quad+\sum_{i}\left(\nabla_{e_{i}} \varphi\right)\left(R(I X, Y) I e_{i}\right)=\frac{1}{2} \sum_{i}\left(\nabla_{e_{i}} \nabla_{e_{i}} D_{\varphi}\right)(X, Y) .
\end{aligned}
$$

Therefore from (3.5), (3.6) and Proposition 3.1, (3) we obtain

$$
\begin{aligned}
\langle\Delta \boldsymbol{u}- & \left.\boldsymbol{u}, \nabla_{X} \nabla_{Y} \boldsymbol{f}\right\rangle \\
= & -\frac{1}{2} \sum_{i}\left(\nabla_{e_{i}} \nabla_{e_{i}} D \varphi\right)(X, Y)+\frac{1}{2} \sum_{i}(d \varphi)\left(e_{i}, R(I X, Y) I e_{i}\right) \\
& +\sum_{i}\left(\nabla_{e_{i}} \varphi\right)\left(R(I X, Y) I e_{i}\right)-\frac{1}{2}(D \varphi)(X, Y) .
\end{aligned}
$$

We have

$$
\begin{aligned}
\sum_{i}( & \left.\nabla_{e_{i}} \nabla_{e_{i}} D \varphi\right)(X, Y) \\
& =D\left(\sum_{i} \nabla_{e_{i}} \nabla_{e_{i}} \varphi\right)(X, Y)+\frac{1}{2}(D \varphi)(X, Y)+2 \sum_{i}(D \varphi)\left(R\left(X, e_{i}\right) Y, e_{i}\right) \\
& =-(D \Delta \varphi)(X, Y)+(D \varphi)(X, Y)+2 \sum_{i}(D \varphi)\left(R\left(X, e_{i}\right) Y, e_{i}\right)
\end{aligned}
$$

Thus we have proved the equality:

$$
\begin{aligned}
\langle\Delta \boldsymbol{u}- & \left.\boldsymbol{u}, \nabla_{X} \nabla_{Y} \boldsymbol{f}\right\rangle \\
= & \frac{1}{2}(D \Delta \varphi)(X, Y)-(D \varphi)(X, Y)+\frac{1}{2} \sum_{i}(d \varphi)\left(e_{i}, R(I X, Y) I e_{i}\right) \\
& +\sum_{i}\left(\nabla_{e_{i}} \varphi\right)\left(R(I X, Y) I e_{i}\right)-\sum_{i}\left(D_{\varphi}\right)\left(R\left(X, e_{i}\right) Y, e_{i}\right) .
\end{aligned}
$$

Since $A=R(I X, Y) I$ is symmetric with respect to $\nu$, we have

$$
\sum_{i}(d \varphi)\left(e_{i}, R(I X, Y) I e_{i}\right)=0
$$




$$
\begin{aligned}
& \sum_{i}\left(\nabla_{e_{i}} \varphi\right)\left(R(I X, Y) I e_{i}\right)-\sum_{i}(D \varphi)\left(R\left(X, e_{i}\right) Y, e_{i}\right) \\
& \quad=\frac{1}{2} \sum_{i}(D \varphi)\left(e_{i}, R(I X, Y) I e_{i}\right)-\sum_{i}(D \varphi)\left(R\left(X, e_{i}\right) Y, e_{i}\right) .
\end{aligned}
$$

By Lemma 3.12 below the right hand side of the last equality is equal to $-\sum_{i}\left(L_{0} \varphi\right)\left(R\left(Y, e_{i}\right) X, e_{i}\right)$. We have thereby proved (2).

Lemma 3.12. For $\theta \in \Gamma\left(S^{2} T^{*}\right)$ we have the equality:

$$
\begin{aligned}
\sum_{i} \theta( & \left.R(I X, Y) I e_{i}, e_{i}\right)-2 \sum_{i} \theta\left(R\left(X, e_{i}\right) Y, e_{i}\right) \\
= & 2 \sum_{i} \theta^{\prime}\left(R\left(e_{i}, Y\right) X, e_{i}\right)
\end{aligned}
$$

where $\theta^{\prime}$ is the anti-hermitian part of $\theta$.

This is a lemma in general Kählerian manifolds generalizing Proposition 4.5 in [3] and can be proved just in the same way as that proposition.

V. We know that a solusion of the equation $L \varphi=0$ is also a solution of the equation $L_{0} \varphi=0$. Hence by Lemmas 3.7 and 3.10 every solution $\varphi$ of the equation $L \varphi=0$ can be uniquely written as

$$
\varphi=d f+\varphi_{1}
$$

where $f$ is a solution of equation (3.4) and $\varphi_{1}$ is a solution of the equations

$$
L \varphi=\delta \varphi=0
$$

Therefore by Theorem 1.2, Proposition 2.2 and Lemma 3.8 we arrive at the following conclusion: In order to prove $\rho(\boldsymbol{f})=\frac{1}{2} m(m+1)$, it is sufficient to show that, for any solution $\varphi$ of equations (3.7), there is $A \in \mathfrak{D}(m)$ such that $\varphi=\langle\boldsymbol{A} \boldsymbol{f}, d \boldsymbol{f}\rangle$.

This being said, let $\varphi$ be a solution of equations (3.7) and $\boldsymbol{u}$ the corresponding solution of the equation $\Phi_{* f}(\boldsymbol{u})=0$. (The correspondence is given by Theorem 1.2.) Then our task is to show that there is $A \in \mathfrak{D}(m)$ such that $\boldsymbol{u}=A \boldsymbol{f}$.

LEMMA 3.13. If we put $\boldsymbol{u}=\left(u_{1}, \cdots, u_{m}\right)$ then the functions $u_{1}, \cdots, u_{m}$ are solutions of equation (3.4).

Proof. By Lemma 3.11, (1) and the fact that $\delta \varphi=0$, we obtain

$$
\left\langle\Delta u-u, \nabla_{X} f\right\rangle=0 \text {. }
$$


Since $\varphi$ satisfies equation (3.3), we have

$$
\Delta \varphi=(d \delta(\varphi I)) I+2 \varphi .
$$

Since $\Delta(\varphi I)=(\Delta \varphi) I$, it follows that $\Delta(\varphi I)=-d \delta(\varphi I)+2 \varphi I$ and hence that $f=\delta(\varphi I)$ is a solution of equation (3.4). Therefore $(d f) I$ is a Killing form by Lemma 3.8 and hence from (3.9) we get $D \Delta \varphi=2 D \varphi$. Consequently by Lemma 3.11, (2) we obtain

$$
\left\langle\Delta \boldsymbol{u}-\boldsymbol{u}, \nabla_{X} \nabla_{Y} \boldsymbol{f}\right\rangle=0 .
$$

Since, at each $p \in M$, the vectors $\nabla_{X} f, \nabla_{X} \nabla_{Y} f\left(X, Y \in T_{p}\right)$ span the vector space $g$, it follows from (3.8) and (3.10) that $\Delta \boldsymbol{u}=\boldsymbol{u}$, proving Lemma 3.13 .

By Lemmas 3.9 and 3.13 we see that $u_{i}$ are linear combinations of $f_{1}, \cdots, f_{m}$. Therefore there is a matrix $A$ of degree $m$ such that $\boldsymbol{u}=A \boldsymbol{f}$.

LEMMA 3.14. $A$ is a skew-symmetric matrix i.e., $A \in \mathfrak{D}(m)$.

Proof. If we put $\boldsymbol{u}^{\prime}=-{ }^{t} A \boldsymbol{f}$, then we have

$$
\begin{aligned}
\left\langle d \boldsymbol{f}, d \boldsymbol{u}^{\prime}\right\rangle & =-\left\langle d \boldsymbol{f},{ }^{t} A d \boldsymbol{f}\right\rangle \\
& =-\langle A d \boldsymbol{f}, d \boldsymbol{f}\rangle \\
& =-\langle d \boldsymbol{u}, d \boldsymbol{f}\rangle=0,
\end{aligned}
$$

meaning that $\boldsymbol{u}^{\prime}$ is a solution of the equation $\Phi_{* f}(\boldsymbol{u})=0$. We now show that the solution $\boldsymbol{u}^{\prime}$ just corresponds to the given solution $\varphi$ of the equation $L \varphi=0$. Indeed by (3.5) and Lemma 3.9 we have $\langle\boldsymbol{u}, \boldsymbol{f}\rangle=\langle\boldsymbol{u}, \Delta \boldsymbol{f}\rangle$ $=\delta \varphi=0$. Hence we have

$$
\begin{aligned}
\left\langle\boldsymbol{u}^{\prime}, \nabla_{X} \boldsymbol{f}\right\rangle & =-\left\langle{ }^{t} A \boldsymbol{f}, \nabla_{X} \boldsymbol{f}\right\rangle=-\left\langle\boldsymbol{f}, \nabla_{X} \boldsymbol{u}\right\rangle \\
& =-\nabla_{X}\langle\boldsymbol{f}, \boldsymbol{u}\rangle+\left\langle\nabla_{X} \boldsymbol{f}, \boldsymbol{u}\right\rangle \\
& =\varphi(X)
\end{aligned}
$$

i.e., $\left\langle\boldsymbol{u}^{\prime}, d \boldsymbol{f}\right\rangle=\varphi$, proving our assertion. Therefore we have $\boldsymbol{u}^{\prime}=\boldsymbol{u}$ and hence $\left(A+{ }^{t} A\right) f=0$, from which follows that $A+{ }^{t} A=0$ (cf. Proof of Proposition 2.1).

We have thus completed proof of Theorem 3.4 .

\section{APPENDIX}

In this appendix we shall accomplish the proof of Theorem 2.3, as we promised. 
We first introduce inner products $\langle$,$\rangle in the vector bundles T^{*}$ and $F$. We define an inner product (, ) in $\Gamma\left(T^{*}\right)$ by

$$
\left(\varphi, \varphi^{\prime}\right)=\int_{M}\left\langle\varphi, \varphi^{\prime}\right\rangle d v
$$

for all $\varphi, \varphi^{\prime} \in \Gamma\left(T^{*}\right)$, where $d v$ is the volume density associated with some Riemannian metric on $M$, and define a norm \|\| in $\Gamma\left(T^{*}\right)$ by $\|\varphi\|^{2}=(\varphi, \varphi)$. In the same way we define an inner product (, ) and a norm \|\| in $\Gamma(F)$. We also introduce a Sobolev norm \|\|$_{1}$ in $\Gamma\left(T^{*}\right)$.

For $f \in V\left(f_{0}\right)$, let $L^{f *}$ be the adjoint operator of $L^{f}$ with respect to the inner products (, ). Since $L^{f}$ differentiably depends on $j^{2} f$, it follows that the operator $\square^{f}=L^{f *} L^{f}$ differentiably depends on the 3-jet $j^{3} f$ of $f$ in an analogous sense. We also note that $\square^{f}$ is strongly elliptic, because $L^{f}$ is elliptic.

LEMMA A. There is a neighborhood $V_{1}\left(f_{0}\right)$ of $f_{0}$ in $\mathfrak{E}(M, m)_{C^{2}}$ such that

$$
\|\varphi\|_{1}^{2} \leqq C\left(\left\|L^{f} \varphi\right\|^{2}+\|\varphi\|^{2}\right)
$$

for all $\varphi \in \Gamma\left(T^{*}\right)$ and $f \in V_{1}\left(f_{0}\right)$, where $C$ is a positive constant independent of $\varphi$ and $f$.

Proof. Since $\square^{f_{0}}$ is strongly elliptic, we have the Gärding inequality :

$$
\|\varphi\|_{1}^{2} \leqq C\left(\left\|L^{f_{0}} \varphi\right\|^{2}+\|\varphi\|^{2}\right)
$$

for all $\varphi \in \Gamma\left(T^{*}\right)$, where $C$ is a positive constant independent of $\varphi$. Since $L^{f}$ differentiably depends on $j^{2} f$, we have: For any $\varepsilon>0$, there is a neighborhood $V^{e}\left(\boldsymbol{f}_{0}\right)$ of $\boldsymbol{f}_{0}$ in $\mathfrak{S}(M, m)_{C^{2}}$ such that

$$
\left\|L^{f} \varphi-L^{f_{0}} \varphi\right\| \leqq \varepsilon\|\varphi\|_{1}
$$

for all $\varphi \in \Gamma\left(T^{*}\right)$ and $f \in V^{\epsilon}\left(f_{0}\right)$. Lemma A follows easily from these facts.

We are now in position to prove Theorem 2.3. Suppose that Theorem 2.3 is not true. Then there is a sequence $f_{i}(i=1,2, \ldots)$ of elements in $\mathfrak{\&}(M, m)$ such that $\boldsymbol{f}_{i} \rightarrow \boldsymbol{f}_{0}$ in $\mathfrak{E}(M, m)_{C^{3}}$ and $\rho\left(\boldsymbol{f}_{i}\right)>\rho\left(\boldsymbol{f}_{0}\right)$. Hence, for each $i$, we can find $k=\rho\left(f_{0}\right)+1$ elements $\varphi_{1}^{(i)}, \cdots, \varphi_{k}^{(i)}$ in $\Gamma\left(T^{*}\right)$ such that $L^{f_{i}} \varphi_{\lambda}^{(i)}=0$ and $\left(\varphi_{\lambda}^{(i)}, \varphi_{\mu}^{(i)}\right)=\delta_{\lambda \mu}$. By Lemma $\mathrm{A}$ we have $\left\|\varphi_{\lambda}^{(i)}\right\|_{1}^{2} \leqq C$. Therefore by the Rellich lemma, we may assume that, for each $\lambda$, the sequence $\varphi_{\lambda}^{(i)}(i=1,2, \ldots)$ converges to an element $\varphi_{\lambda}$ in the completion of $\Gamma\left(T^{*}\right)$ 
with respect to the norm \|\| . For every $\psi \in \Gamma\left(T^{*}\right)$, we have $\left(\varphi_{i}^{(i)}, \square^{f_{i}} \psi\right)$ $=\left(\square^{f_{i}} \varphi_{i}^{(i)}, \psi\right)=0$. Since $\square^{f}$ differentiably depends on $j^{3} f,\left\|\square^{f_{i}} \psi-\square^{f_{0}} \psi\right\|$ $\rightarrow 0$. It follows that $\left(\varphi_{\lambda}, \square^{f_{0}} \psi\right)=0$. Therefore by the hypoellipticity of $\square^{f_{0}}, \varphi_{\lambda} \in \Gamma\left(T^{*}\right)$ and $L^{f_{0}} \varphi_{\lambda}=0$. Since $\left(\varphi_{\lambda}, \varphi_{\mu}\right)=\delta_{\lambda \mu}$, we have $\rho\left(f_{0}\right) \geqq k=$ $\rho\left(f_{0}\right)+1$, which is a contradiction. We have thus completed proof of Theorem 2.3.

\section{BIBLIOGRAPHY}

[1] J. F. Adams, P. D. Lax and R. S. Phillips, On matrices whose real linear combinations are non-singular, Proc. Amer. Math. Soc., 16 (1965), 318-322.

[2] E. Cartan, Sur la possibilité de plonger un espace riemannien donné dans un espace euclidien, Ann. Soc. Pol. Math., 6 (1927), 1-17.

[ 3 ] S. Kobayashi and K. Nomizu, Foundations of Differential Geometry I, Interscience Publishers, New York, 1963; II, 1969.

[4] K. Kodaira and D. C. Spencer, On deformations of complex analytic structures III, Ann. Math., 71 (1960), 43-76.

[5] A. Lichnerowicz, Géométrie des Groupes de Transformations, Dunod, Paris, 1958.

[6] J. F. Nash, The imbedding problem for Riemannian manifolds, Ann. Math., 63 (1956), 20-63.

[ 7 ] L. Nirenberg, The Weyl and Minkowski problems in the differential geometry in the large, Comm. Pure and Appl. Math., 6 (1953), 337-394.

[ 8 ] D. G. Quillen, Formal properties of over-determined systems of linear partial differential equations, Ph. D. thesis, Harvard University, 1964.

[ 9 ] I. M. Singer and S. Sternberg, The infinite group of Lie and Cartan, J. Analyse Math., 15 (1965) , 1-114.

[10] K. Shiga and R. Sunada, The Hodge Decomposition in Elliptic Complexes, Seminar Reports of Global Analysis, Tokyo Institute of Technology, 1971 (in Japanese).

[11] N. Tanaka, Rigidity for elliptic isometric imbeddings, Proc. Japan Acad., 48 (1972), 370-372.

[12] K. Yano, Differential Geometry on Complex and Almost Complex Spaces, Pergamon Press, New York, 1965.

Kyoto University 